\title{
Lithium Depletions in Late-type Dwarfs
}

\author{
D. R. Xiong ${ }^{1}$ and L. Deng ${ }^{2}$ \\ ${ }^{1}$ Purple Mountain Observatory, Chinese Academy of Sciences,Nanjing 210008 \\ email: xiongdr@pmo.ac.cn \\ ${ }^{2}$ National Astronomical Observatories, Chinese Academy of Sciences, Beijing 100012 \\ email: licai@bao.ac.cn
}

\begin{abstract}
Using a self-consistant dynamic theory of non-local convection in chemically inhomogeneous stars, the lithium depletion in MS stars of masses $0.725-1.5 M_{\odot}$ are calculated. Both of the overshooting and microdiffusion are included in a consistent way. The comparisons of theretical reasults with the observed Li depletions in open clusters show that the general characters of Li depletions can be reproduced by theory. The overshooting mixing and microdiffusion induced by gravitational setting and radiative accelerations may be two main mechanisms of Li depletion.
\end{abstract}

Keywords. Convection- star: abundance-star: late-type-stars:evolution- open clusters and associations: general

It is well known that the $\mathrm{Li}$ abundance is very significant for the theory of big-bang nucleosynthesis and tracking the extension of the surface convection zone in stars during the course of their evolution.

The mechanism of Li depletion is still not completely known. A number of theoretical approach has been presented so far, such as the mass loss (Weymann \& Sears, 1965; Schramm et al., 1990), wave-driven mixing (Garcia López \& Spruit, 1991; Montalban \& Schatzman, 1996), rotationally induced mixing (Charboneau et al., 1992; Chaboyer et al., 1995; Pinsonneault, et al., 1999), microdiffusion and turbulent mixing (Michaud, 1986; Turcotte et al., 1998), overshooting mixing (Straus et al., 1976; Xiong \& Deng, 2001) and so on. However, there is no any one single mechanism which can interpret all the known observed characters of Li depletion. The Li depletion, in our opinion, shoult be the result of several physical processes, rather than from a single mechanism. The overshooting mixing and microdiffusion induced by gravitation setting and ratiation acceleration seem to be a reasonable combination.

Using our non-local convection theory in chamically inhomogeous stars (Xiong, 1981), we calculated the Li depletions for 20 series of evolutionary models with mass of 0.725-1.5 $M_{\odot}$. The solar abundance $(\mathrm{X}=0.70, \mathrm{Z}=0.02)$ is assumpted. The overshooting mixing and microdiffusion induced by gravitational setting are included in a selfconsistant way. The fundamental equations and the numerical scheme will be described in our latter paper (Xiong \& Deng, 2008). Figure 1 illustrates the Li abundances as function of stellar age for the evolutionary models with different masses as marked on the curves. In Fig. 1a the microdiffusion is neglected. In Fig. 1b both of the microdiffusion and overshooting mixing are included. It can be seen that the Li abundances decrease (approximately) exponnentially with age of stars. The e-folding time of Li depletion are not a monotonic function of stellar mass. It achives the maximum at $M \approx 1.1 M_{\odot}$. This is due to the fact that the Li depletion is resulted dominantly from macrodiffusion for warm stars $\left(M \geqslant 1.1 M_{\odot}\right.$ or $\left.T e \geqslant 6100 K\right)$, and it is resulted dominantly from the overshooting mixing for the cool stars $\left(M \leqslant 1.0 M_{\odot}\right.$ or $\left.T e \leqslant 5800 K\right)$. The sign $\odot$ in Figs. $1 \& 3 \mathrm{~d}$ is 

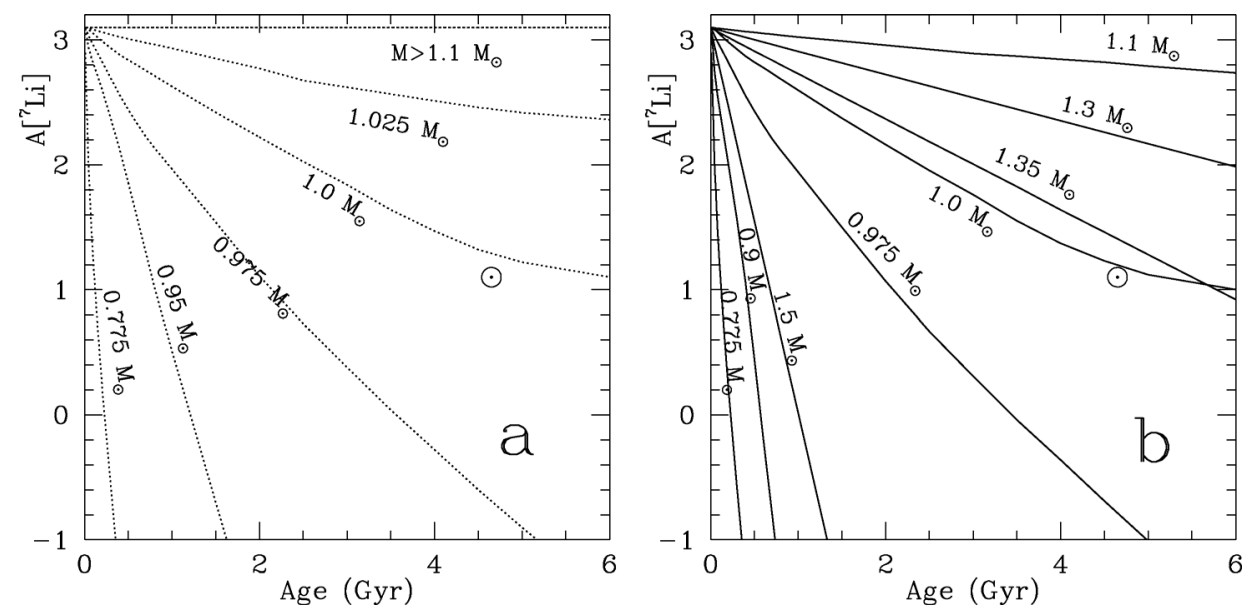

Figure 1. The Li abundances as functions of star's age for the different masses marked on the curves. (a) the microdiffusion is neglected, (b) both of the microdiffusion and overshooting mixing are included.

the observed lacation of the Sun. It is necessary to indicate that the depth of our solar model agrees with the requirement of helioseismology.

Figs. 2a $-\mathrm{d}$ show the lithium distribution in stellar interior at different ages. The short dashed lines mark the bottom boundary of convectively unstable zone. The sharply drop at bottom is due to violent burn of lithium. It can be seen that the convective overshooting (the shadowy regions) penetrates deeply into the convectively stable zone and the full mixiong zone is extended greatly. For the warm stars with mass greater than abut $1.1 M_{\odot}$ (Figs. $2 \mathrm{a} \& \mathrm{~b}$ ), the convection zone is too shallow. The gravitational setting is not enough to bring the surface lithium to the deep burn region. It drive the surface lithium to the deeper interior of stars and the surface lithium will be stored in the lower radiative region under the overshooting zone, so the surface lithium abundance decreases. The convection zone becomes deeper with decrease of stellar temperature. overshooting brings the surface lithium to the deep burn region, therefore, the surface lithium abundance decreases (Figs $2 c \& d$ ). We can know from the above analysis that the mechanisms of lithium depletion are different for the warm and cool stars. For the warm stars the microdiffusion is domanial, however the oversooting mixing becomes domanial for the cool stars.

Figure 3 give The Li abundance vs. the effect temperature for the clusters with different ages, and the corresponding theoretical isochne lines of $\mathrm{Li}$ abundance are also drawn here. It can be seen from Fig. 3 that within the observational uncertain the theoretical results reproduced roughly the general profiles of Li depletion except the following points: (1) The Li depletions seem to be underestimated for stars arroud $T e \approx 5900 \mathrm{~K}$. It is possible there are depletion mechanisms ignored in this work; (2) In Fig. 3d the Li abundances of five stars on the right of theoretical isochne line seem to be too high. However, two of them are only given the upper limits of their Li abundance. The another two stars, named as I-2a and I-2b in the Table 1 of Jones et al. (1999), are the two components of a double-lined spectroscopic binary. Their Li abundance are quite uncertain. The samples of lower temperature star are too few for giving a confident conclution.

It is the conclusion that the overshooting mixing and microdiffusions induced by gravitation setting and radiative acceleration may be two main mechanisms of Li depletion. Not only the convective overshooting interpret the lithium depletion of cool stars but 

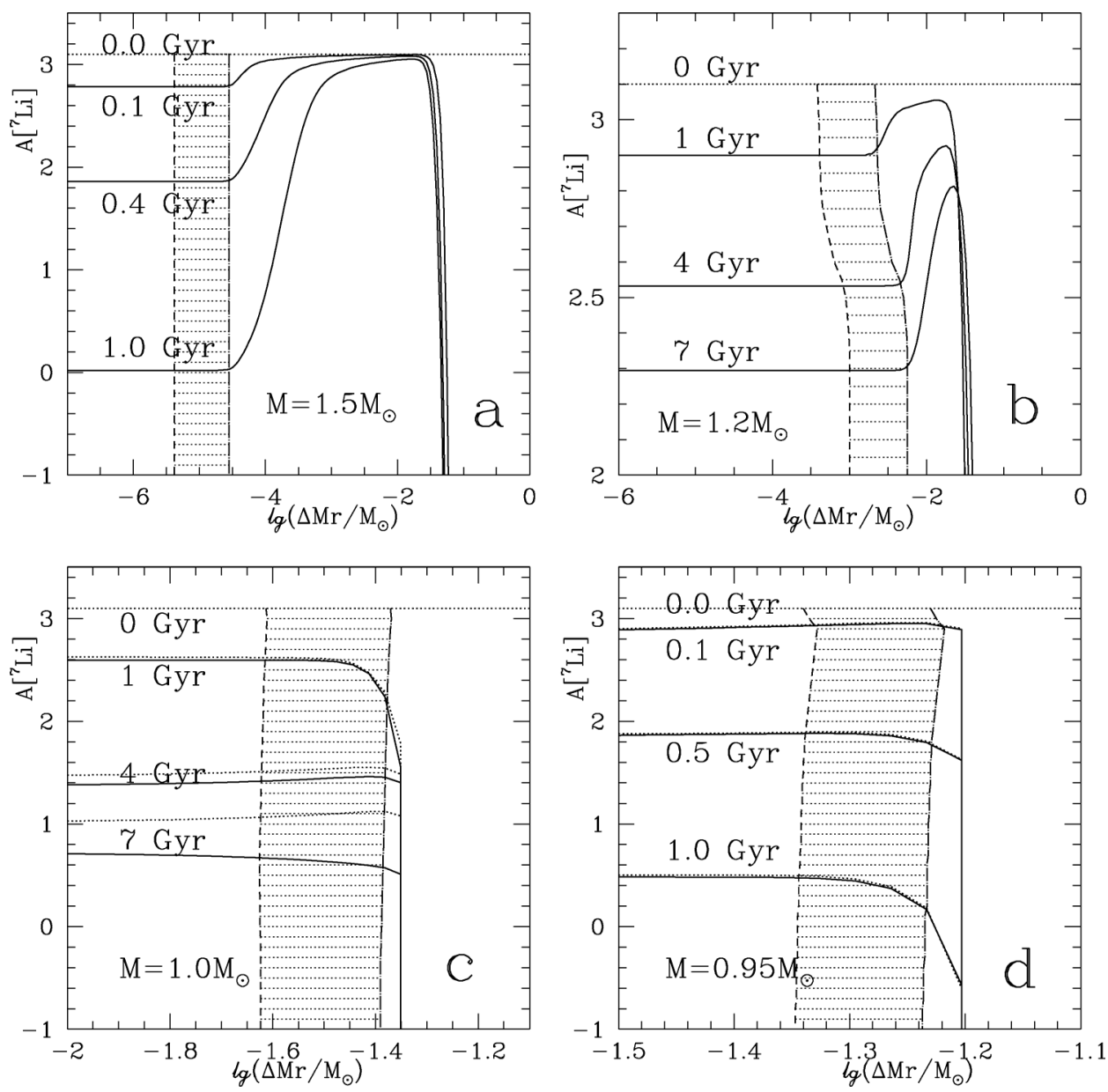

Figure 2. The lithium distribution in stellar interior at different ages, (a) $M=1.5 M_{\odot}$ (b) $M=1.2 M_{\odot}\left(\right.$ c) $M=1.0 M_{\odot}(\mathrm{d}) M=0.95 M_{\odot}$

also it increase the mass of the surface full mixing region, therefore the too quiuck depletion of lithium in warm stars predicted by single microdiffusion theory is removed automatically. It is not necessary to induce a phenomenological turbulent diffusion such as induced by Proffitt \& Michaut (1991) and Richard, Michaud \& Richer (2005). In other words, convective overshootng is just the so called turbulet diffusion needed by theim.

\section{Acknowledgements}

This work is supported in part by NSFC through grants 10773029 and 10778719 .

\section{References}

Chaboyer, B., Demarque, P., \& Pinsonneault, M.H., 1995, ApJ 441, 865

Charboneau, P., Vauclair, S., \& Zahn, J. P., 1992 A\&GA, 255, 191

Garcia López, R.J. \& Spruit, H. C., 1991 ApJ, 377, 268

Jones, B. F., Fischer, D., \& Soderblom, D. R., 1999 AJ, 117, 330

Michaud, G., 1986 ApJ, 302, 650

Montalban, J. \& Schtzman, E., 1996 [5 A\&A, 305,513

Pinsonneault, M. H., Walker, T. P., Steigman, G., \& Narayanan, V. K., 1999 ApJ, 527, 180 

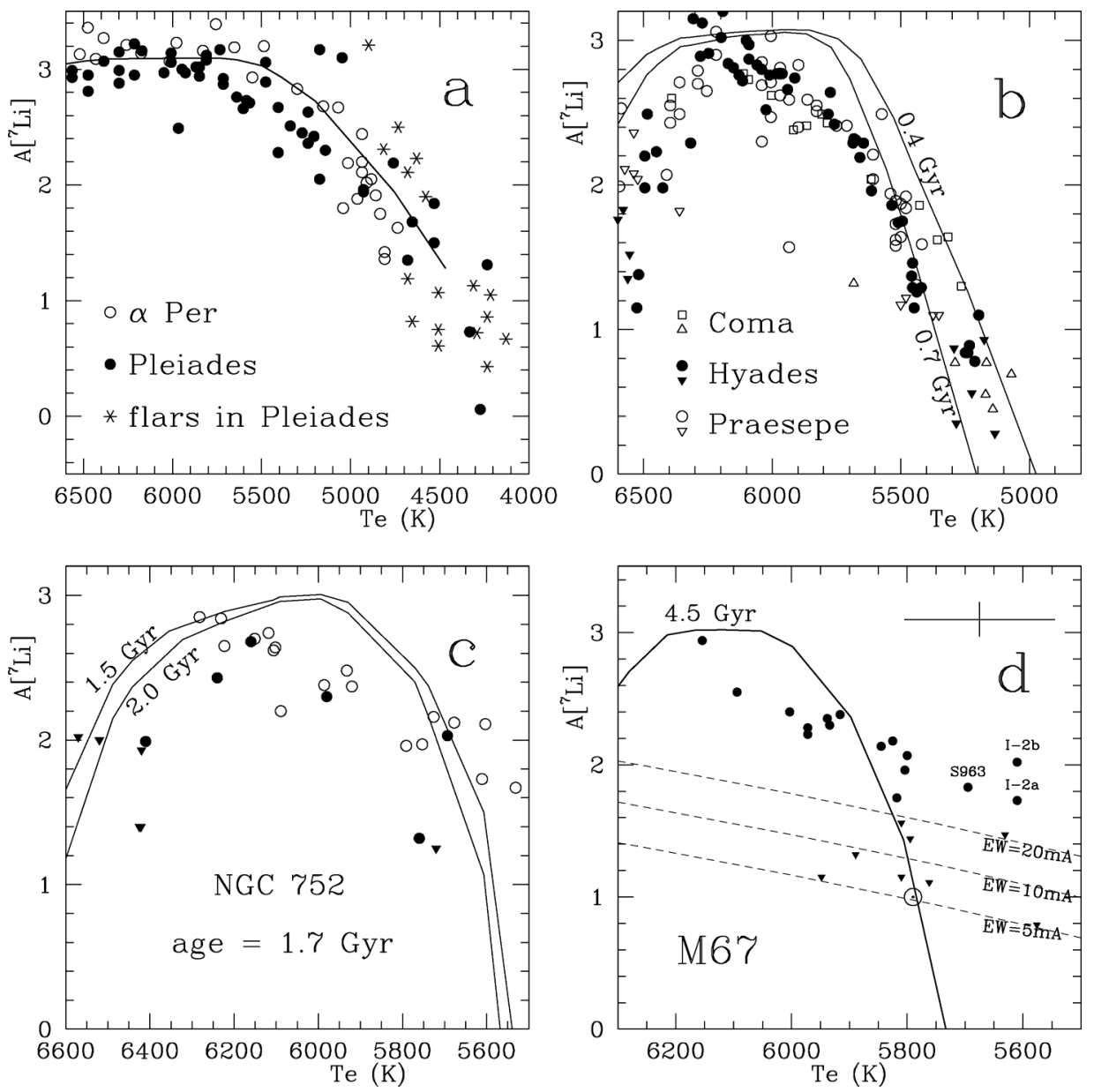

Figure 3. The Li abundances versus effective temperature of stars for (a) $\alpha$ Persei, Pleiades, (b) Coma Berenices, Hyades Prasepe, (c) NGC 752, and (d) M67 clusters. The triangles and inverse triangles give the upper limits for Li abundance. The estimated uncertainties of LI abundance and effect temperature are indicated in the upper-right corner of the plots.

Proffitt, C. R. \& Michaud, G. 1991 ApJ, 380, 238

Richard, O., Michaud, G., \& Richer, J., 2005 ApJ, 619, 538

Schramm, D. N., Steigman, G., \& Dearborn, D. S. P., 1990 ApJ, 359, L55

Straus, J. M., Blake, J. B., \& Schramm, D. N., 1976 ApJ, 204, 481

Turcotte, S., Richer, J., \& Michaud, G., 1998 ApJ, 504, 559

Weymann, R. \& Sears, R. L., 1965 ApJ, 142, 174

Xiong, D. R., 1981 Scientia Sinica, 24,1406

Xiong, D. R. \& Deng, L. 2001 MNRAS, 327, 1137

Xiong, D. R. \& Deng, L. 2008 MNRAS, prepared

\section{Discussion}

AsPlund: Have you investigated how much ${ }^{7} \mathrm{Li}$ depletion your convective overshooting model predict for metal-poor turn-off stars?

XIONG: We have only considered the depletion of Lithium for solar type stars. We understand that Lithium depletion in metal-poor stars is extremely important in exploring 
the initial cosmological Lithium abundance and nucleosynthesis of Big-Bang. Due to the lack of reliable evolutionary models of metal-poor stars, such work can only be done in a future time when such models become available.

LANGER: Are there any free parameters in your model for overshooting? and: Are your models (for main sequence stars) in strict thermal equilibrium?

XIONG: 1). Indeed, there is also a free parameter in our statistical theory of non-local turbulent convection, which is similar to that of MLT representing the characteristic length (wave number) of the turbulent spectrum. However, such a parameter is not at all "free". Instead, it has to be regulated by observational constraints from stellar evolution, depletion of Lithium in stars, helioseismology and stellar pulsation, etc. As we have shown in our previous work, the theory stands against all these challenges.

2 ). The models computed so far are all located on the main sequence, therefore the assumption of perfect thermal equilibrium is reasonable.

KuPKA: Have you also made any computations for the Li abundance in hotter (main sequence) stars than the ones you have shown here? I mean early $\mathrm{F}$ type and late A type, and compared to some data? This could be useful to test the model for microscopic diffusion you have used in your computations in the OV zone of cool main sequence stars too!

XIONG: We have done Lithium depletion models only for late $\mathrm{F}$ type $-\mathrm{K}$ type stars so far. The late A type - early F type stars are not modelled, mainly because we could not good monochromatic opacity. For microdiffussion, only gravitational settling has been taken into account. Radiative acceleration has been excluded. Once monochromatic opacity is available, radiative acceleration can be included into microdiffusion easily. In that case, the combination of microdiffusion and overshooting mixing can be well applied to the studies of $\mathrm{Li}$ depletion and $\mathrm{A}_{p}, \mathrm{~F}_{m}$ phenomena in A-F type stars. Turbulent diffusion is very likely not needed, pretty the same way as in the present work that overshooting can naturally reproduce the effect of turbulent diffusion 